\title{
Protein-doped droplet beams for laser-aligned serial crystallography.
}

\author{
U. Weierstall, J.C.H. Spence, R.B. Doak, D. Starodub \\ Department of Physics and Astronomy, Arizona State University, Tempe, AZ 85287-1504
}

Research in cryoelectron microscopy has established that diffraction from at least $10^{4}$ proteins is needed to produce a statistically significant three-dimensional reconstructed charge-density map at $0.3 \mathrm{~nm}$ resolution. Electron (or X-ray) diffraction from a beam of laser-aligned proteins has been proposed as an approach to structure determination of proteins, which cannot be crystallized [1]. In our method a transmission diffraction pattern builds up by accumulating patterns from successive oriented identical single molecules in a molecular beam. If at any instant there are $\mathrm{N}$ identical molecules in the beam, the recording time is reduced by $\mathrm{N}$ over that for a single molecule, where $\mathrm{N}$ is the number of ice-coated molecules. Thus, apart from the "coherent amplification" effects, which occur in crystallography, our arrangement is similar to diffraction from a stationary crystal of $\mathrm{N}$ molecules. The protein injection source produces a jet of micron sized water droplets, each containing one hydrated protein or macromolecular complex. The molecules pass in vacuum across a focused, high-energy electron or X-ray beam, where they are also immersed in a polarized focused laser beam for molecular alignment. The degree of alignment depends on temperature and laser power [2]. A two-dimensional diffraction pattern accumulates continuously at the detector for a fixed laser polarization and molecular orientation before being read out. Repeating this process for many orientations would fill 3D reciprocal space and would allow 3D reconstruction of the molecule by iterative phase retrieval methods [3]. In order to minimize the background contribution to the electron diffraction pattern from water scattering and to enable laser alignment, surplus water must be removed by evaporation leaving all but a thin water layer. It has been shown by electrospray TOF spectroscopy [4] that the removal of essentially all "bulk" water does not cause proteins in the electrospray beam to unfold.

We have evaluated several protein injection methods experimentally. In order to reduce electron diffraction exposure time, it is necessary to obtain a high particle flux at the interaction region (the intersection of alignment laser and electron beam), therefore the droplets should preferably be single file and monodirectional. To ensure one particle per droplet, they should also be monodispersed. Both properties can be achieved with a Rayleigh droplet beam as shown in Fig.1. There, a liquid is forced at high pressure through a small vibrating nozzle forming a jet, which breaks up into droplets to minimize its free surface energy. The droplet diameter is twice the nozzle diameter. Production of droplets smaller than 2 microns by this method is difficult despite filtering due to clogging. Another method uses electro-hydrodynamic droplet generation (electrospray). Highly charged droplets are produced by applying a high voltage between the nozzle and an extraction electrode. Under certain conditions of voltage and pressure, one can obtain a monodispersed, monodirectional beam of droplets in a small area behind the nozzle, before the droplets Coulomb explode. Figure 2 shows both modes of operation in air with the same nozzle of 8 micron diameter. In electrospray mode, much smaller droplets are produced. The droplet images were taken in air and vacuum with 100nsec single flash illumination from a laser diode. Both injection modes will be discussed. Adding a coaxial gas shroud around the nozzle can assist the water removal.

We use e-coli as a test object for measuring alignment in a strong laser field. Its large size $(\sim 2 \mathrm{x}$ 0.5 micron) should make alignment possible at room temperature (large polarizability and induced dipole moment). A stroboscopic image of e-coli doped droplets is shown in Fig.3. The presence of e- 
coli in the droplets was confirmed by examining the water collected on a slide. Similar tests have been done with 250nm $\mathrm{Au}$ balls. We have experimentally confirmed by optical absorption spectroscopy that photosystem 1 protein is not damaged during transport in a Rayleigh droplet beam by the high pressure involved. To improve alignment at lower temperatures (less Brownian angular motion), droplets of cryogenic fluid may be doped with molecules as demonstrated for aminoacids $[5,6]$.

\section{References}

[1] J. C. H. Spence, and R. B. Doak., Phys. Rev. Letts. 92: (2004) Art. No. 198102.

[2] J. C. H. Spence, K. Schmidt, J. S. Wu, G. Hembree, U. Weierstall, B. Doak and P. Fromme, Acta Cryst. A 61: (2005) 237-245

[3] Marchesini, S., H. He, H. N. Chapman, S. P. Hau-Riege, A. Noy, M. R. Howells, U. Weierstall and J. C. H. Spence, Phys. Rev. B 68: (2003) Art. No. 140101.

[4] Ruotolo, B., Giles, K., Campuzano, I., Sandercock, A., Bateman, R., Robinson, C.V. Science Express, Nov 17, (2005) 1120177.

[5] Lindinger, A., J. P. Toennies and A. F. Vilesov, J. Chem. Phys. 110: (1999) 1429-1436.

[6] Supported by NSF SGER award DBI-0429814 and an award from CBST/NSF.
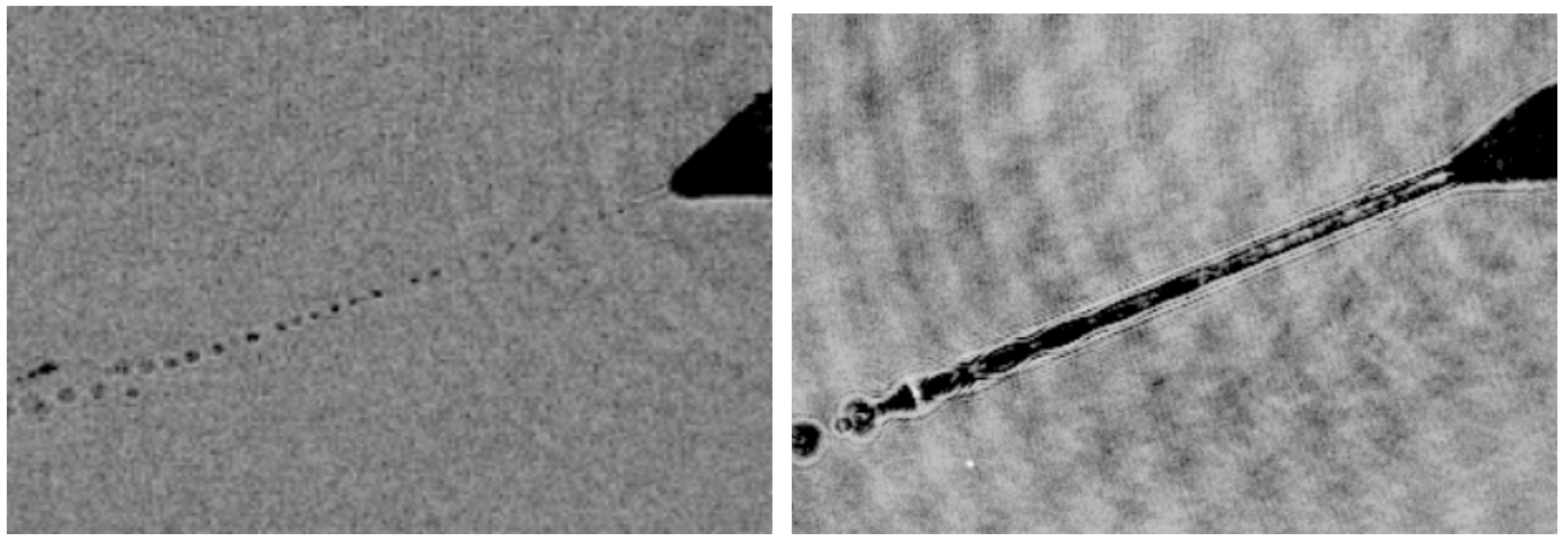

FIG. 1. Comparison of Electrospray (left) and Rayleigh (right) droplet beams from the same 8micron nozzle. The Rayleigh beam is driven with 100psi pressure and triggered by piezo vibrator operating at by a $600 \mathrm{kHz}$ without extraction voltage. ES voltage is $2.6 \mathrm{kV}$. Both images obtained with a single 100ns flash of illumination from a laser diode. The beams are in air.

The much smaller diameter of the ES droplets, drawn from the tip of a liquid cone, are evident.

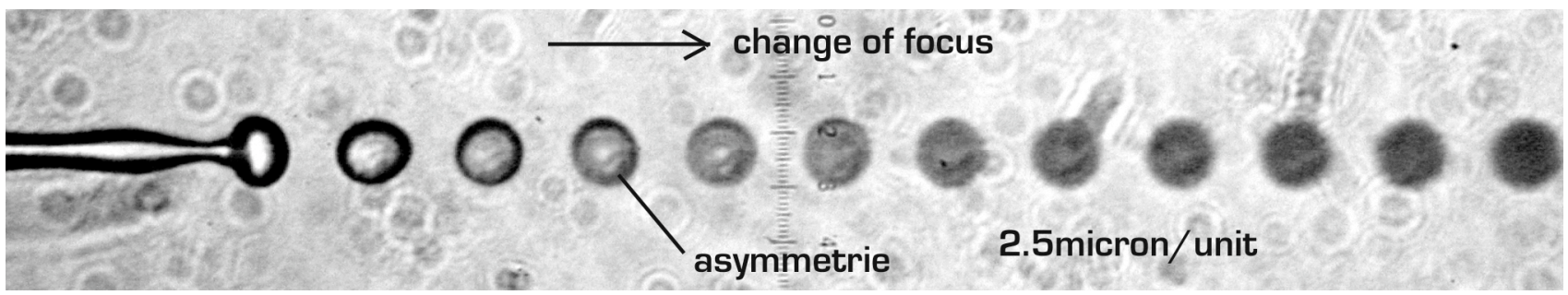

FIG. 2. Rayleigh droplet beam doped with e-coli bacteria. Stroboscopic image synchronized with the droplet breakup frequency. 